\title{
Adesão ao tratamento farmacológico de pacientes em hemodiálise
}

\author{
Adherence to pharmacological treatment in adult patients \\ undergoing hemodialysis
}

\section{Autores}

Vanessa Sgnaolin

Ana Elizabeth Prado Lima Figueiredo

Pontifícia Universidade Católica do Rio Grande do Sul - PUC/RS.

Data de submissão: 27/06/2011 Data de aprovação: 16/11/2011

\footnotetext{
Correspondência para: Vanessa Sgnaolin Rua Antônio Joaquim Mesquita 302/337,Passo D'Areia

Porto Alegre - RS - Brasil CEP 91350-180

E-mail: vanessasgnaolin@ yahoo.com.br

O referido estudo foi realizado na PUC/RS.

Os autores declaram a inexistência de conflitos de interesse.
}

\section{Resumo}

Introdução: A adesão ao tratamento do paciente em terapia hemodialítica não é um processo simples. As estratégias para promover a adesão vão ao encontro da necessidade de melhorias no processo de orientação sobre a doença e o seu tratamento farmacológico. Objetivos: Identificar a adesão ao tratamento farmacológico de pacientes em hemodiálise e os principais fatores relacionados, por meio do uso de uma Escala de Adesão. Métodos: Estudo observacional, descritivo e transversal. Foram realizadas entrevistas para o levantamento de dados socioeconômicos, farmacoterapêuticos e de autorrelato de adesão farmacológica. Resultados: Dos 65 pacientes participantes, $55,4 \%$ demonstraram não adesão. A média de medicamentos utilizados foi de 4,1 $\pm 2,5$ (autorrelato) e 6,2 $\pm 3,0$ (prescrição). A análise estatística mostrou diferença significativa entre adesão e idade em diferentes faixas etárias ( $\geq 60$ anos apresentaram maior adesão). Conclusões: Uma proporção significativa dos pacientes tem dificuldades para aderir ao tratamento e o principal fator citado foi o esquecimento. Em relação à idade, os pacientes idosos se mostraram mais aderentes. O baixo nível de conhecimento sobre os medicamentos utilizados pode ser um dos motivos da má adesão, sendo o processo de orientação do paciente por parte da equipe de profissionais envolvidos na assistência uma estratégia para promover a adesão.

Palavras-chave: Adesão à medicação. Atenção farmacêutica. Unidades hospitalares de hemodiálise.

\section{Abstract}

Introduction: Adherence to treatment in patients on hemodialysis is not a simple process. Strategies to promote adherence will meet the need for improvements in the process of orientation concerning the disease and its pharmacological treatment. Objectives: To identify compliance with pharmacological treatment of patients on hemodialysis and the main factors related to it we used the Adherence Scale. Methods: Observational, descriptive and cross-sectional study. Interviews were conducted to collect socioeconomic, pharmacological data, as well as those regarding self-reported adherence to drug. Results: Out of the 65 participants, $55.4 \%$ showed non-compliance. The mean number of drugs used was $4.1 \pm 2.5$ (self-report) and $6.2 \pm 3.0$ (prescription). Statistical analysis showed significant differences concerning compliance at different ages ( $\geq 60$ years are more adherent). Conclusions: A significant proportion of patients have difficulty to comply with treatment and the main factor was forgetfulness. Regarding age, elderly patients are more adherent to treatment. The low level of knowledge about the used drugs may be one of the reasons for the lack of adherence, and the patient's orientation process by a team of multiprofessionals involved in assisting is a strategy to promote adherence.

Keywords: Medication adherence. Pharmaceutical care. Hemodialysis units, hospital. 


\section{INTRODUÇ̃̃O}

A Doença Renal Crônica (DRC) é progressiva e de elevada morbimortalidade e, em sua fase avançada, necessita de tratamento de substituição da função renal (TRS). No Brasil, a forma mais comum de TRS é a hemodiálise (HD), e esta deve ser concomitante ao tratamento farmacológico, dietoterápico, entre outros. ${ }^{1}$

A adesão ao tratamento e às orientações dietéticas associados à HD é importante e requer entendimento e aceitação da doença. ${ }^{2,3} \mathrm{~A}$ adesão ao tratamento tem efeito positivo na manutenção da saúde, na qualidade de vida e sobrevida. ${ }^{4,5} \mathrm{O}$ conceito de adesão é dinâmico, complexo, multidimensional e mede a resposta do paciente às recomendações do prescritor. ${ }^{6,7}$

Alguns estudos demonstraram que o regime terapêutico da DRC (dieta, ingesta de líquidos, medicamentos e terapia dialítica) apresenta taxas elevadas de não adesão. ${ }^{8}$ Fatores como: idade, gênero, tabagismo, duração da HD e comorbidades determinam o processo da má adesão. ${ }^{9}$ A percepção do paciente de melhora de saúde, a negação de realizar tratamento prolongado, bem como a complexidade terapêutica e a confusão das orientações também alteram a adesão à terapia. ${ }^{5}$ Dessa forma, se os parâmetros citados forem avaliados pode-se considerar que todos os pacientes são não aderentes. ${ }^{10}$

Desse modo, a avaliação regular da prevalência dos pacientes aderentes ao tratamento é importante. ${ }^{11}$ O objetivo deste trabalho foi identificar a adesão ao tratamento farmacológico do paciente em HD por meio do uso de Escala de Adesão, assim como avaliar os principais fatores que influenciam esse processo. ${ }^{12}$

\section{Métodos}

Estudo observacional, descritivo e transversal. Foram incluídos todos os pacientes em terapia hemodialítica por mais de 3 meses, maiores de 18 anos, que estavam em acompanhamento pelo Serviço de Nefrologia de um Hospital Universitário e que aceitaram participar do estudo por meio da assinatura do Termo de Consentimento Livre e Esclarecido.

A coleta de dados ocorreu no período de março a maio de 2009, por meio de entrevistas individuais durante a sessão de HD. As entrevistas foram realizadas com os pacientes independentemente de terem ou não o suporte de um cuidador, mas sem a interferência destes, pois o objetivo do estudo foi analisar o grau de conhecimento e de comprometimento do próprio paciente sobre a sua terapia farmacológica. Para tanto, foram utilizados os seguintes instrumentos: ficha para levantamento de dados socioeconômicos (sexo, idade, estado civil, cor, escolaridade, profissão, nível salarial, número de moradores no domicílio); perfil farmacoterapêutico (nome do medicamento, dose, posologia, duração do tratamento e indicação terapêutica) e questionário de autorrelato para levantamento da adesão à terapêutica, composto por quatro perguntas fechadas. ${ }^{12}$

Para esse último questionário, as respostas eram pontuadas em sim ou não e atribuído o valor de zero para cada resposta afirmativa (em relação à adesão) e um para as negativas (em relação à não adesão). Escore zero indicava máxima adesão, de um a dois, moderada e de três a quatro, baixa adesão. ${ }^{12}$ Utilizou-se o critério também adotado por outros autores: escore zero indicando adesão e, a partir de um, não adesão. ${ }^{13}$

Em prontuário, foram coletados dados referentes à prescrição dos medicamentos, diagnóstico etiológico, tempo em diálise, registros de não adesão ao tratamento farmacológico e os exames clínicolaboratoriais (ganho de peso interdialítico, pressão arterial pré-diálise, exames laboratoriais de fósforo, cálcio e potássio) realizados na data mais próxima da entrevista.

As amostras de sangue foram encaminhadas ao Laboratório de Patologia Clínica do Hospital. Os valores de referência foram considerados de acordo com as diretrizes da Sociedade Brasileira de Nefrologia (SBN), para o potássio $\leq 5,5 \mathrm{mEq} / \mathrm{L}$, fósforo $\leq 5,5 \mathrm{mg} / \mathrm{dL}$ e produto cálcio $\mathrm{X}$ fósforo $\leq 55 .{ }^{14}$

Os valores de pressão arterial (PA) foram coletados no prontuário eletrônico do paciente e correspondem aos da data em que ocorreu a entrevista. Foram considerados adequados valores de $\mathrm{PA}<140 / 90 \mathrm{mmHg}{ }^{15}$

Para a avaliação da variação de peso interdialítico, foram considerados os valores de peso $(\mathrm{kg})$ obtidos no final da sessão que antecedeu a entrevista e o peso inicial do dia da entrevista. Posteriormente, a variação de peso foi comparada ao peso seco do paciente, sendo considerado como adequado um ganho de peso de até $5 \%$ em relação ao peso seco. ${ }^{16}$

Os dados coletados foram processados e tabulados utilizando o programa Statistical Package for the Social Sciences (SPSS) versão 17. As variáveis categóricas foram apresentadas como frequências e percentuais e as variáveis contínuas como média e desvio padrão. O teste Qui-quadrado de independência foi empregado para testar a associação entre as variáveis categóricas, e os resultados considerados significativos quando $\mathrm{p}<0,05$. 


\section{Resultados}

Dos 78 pacientes em tratamento hemodialítico nos meses em que foram realizadas as entrevistas, $65 \mathrm{pa}$ cientes $(83,3 \%)$ preenchiam os critérios de inclusão, 5 pacientes $(6,4 \%)$ estavam a menos de três meses em tratamento, dois pacientes $(2,6 \%)$ recusaram-se a participar, 2 pacientes $(2,6 \%)$ eram menores de 18 anos e outros $4(5,1 \%)$ eram pacientes em trânsito (Tabela 1).

Em relação à classificação da adesão, as respostas obtidas para cada pergunta da Escala de Morisky foram examinadas individualmente e encontram-se descritas na Tabela 2.

Dessa maneira, foi constatado que 29 pacientes $(44,6 \%)$ relataram-se aderentes e $36(55,4 \%)$ em alguma das questões demonstraram não aderência. De acordo com esses dados, o principal motivo da não adesão ao tratamento farmacológico é o esquecimento $(\mathrm{n}=23,35,4 \%)$.

A média de medicamentos utilizados pelos pacientes em tratamento hemodialítico foi de 4,1 $\pm 2,5$ quando o autorrelato foi analisado, variando de, no mínimo, um medicamento, até, no máximo, dez, e a média de 6,2 $\pm 3,0$ quando considerados os dados encontrados na prescrição médica do prontuário eletrônico com o número mínimo de 1 medicamento prescrito e o máximo de 16.

Do total de 65 pacientes, $7(10,8 \%)$ não lembravam de qualquer medicamento que faziam uso, desses seis, tinham cuidadores, que eram os responsáveis pelo tratamento farmacológicos, e um estava hospitalizado. Apenas seis pacientes $(9,2 \%)$ relataram corretamente o nome de todos os medicamentos prescritos e, quando excluíram-se os medicamentos que são administrados durante a sessão de hemodiálise (Eritropoetina, Ferro III e Vitamina C), dez pacientes $(15,4 \%)$ acertaram o nome de todos os medicamentos prescritos. Somente um dos pacientes $(1,5 \%)$ referiu a dose correta de todos os medicamentos utilizados. Dos 26 pacientes $(40,0 \%)$ que tinham cuidadores, 11 eram maiores de 60 anos e todos erraram o nome e a dose dos medicamentos prescritos. Os cuidadores eram representados por: $42,3 \%$ esposa, $30,8 \%$ filho(a), 11,6\% marido, 7,7\% auxiliar de enfermagem, 3,8\% neto(a) e 3,8\% profissional contratado.

Por meio da análise estatística, observou-se que não ocorreu diferença significativa quando comparada à classificação de adesão com as variáveis socioeconômicas (Tabela 3), clínicas e laboratoriais (Tabelas 4 e 5), exceto quando analisados a idade, na qual os pacientes idosos demonstraram-se mais

\section{Tabela 1 CARACTERISTICAS SOCIOECONÔMICAS DA POPULAÇÃO $(n=65)$}

\begin{tabular}{|c|c|c|}
\hline Variáveis & $\mathrm{n}$ & $\%$ \\
\hline \multicolumn{3}{|l|}{ Sexo } \\
\hline Feminino & 33 & 50,8 \\
\hline Masculino & 32 & 49,2 \\
\hline \multicolumn{3}{|l|}{ Estado civil } \\
\hline Solteiro(a) & 10 & 15,4 \\
\hline Casado(a) & 35 & 53,8 \\
\hline Viúvo(a) & 9 & 13,9 \\
\hline Divorciado(a) & 11 & 16,9 \\
\hline \multicolumn{3}{|l|}{ Trabalho } \\
\hline Sim & 59 & 90,8 \\
\hline Não & 6 & 9,2 \\
\hline \multicolumn{3}{|l|}{ Profissão } \\
\hline Aposentado & 59 & 90,8 \\
\hline Comerciante & 1 & 1,5 \\
\hline Empreiteiro de obra & 1 & 1,5 \\
\hline Professor & 1 & 1,5 \\
\hline Religioso & 1 & 1,5 \\
\hline Telefonista & 2 & 3,2 \\
\hline \multicolumn{3}{|l|}{ Cor } \\
\hline Branca & 34 & 52,3 \\
\hline Parda & 12 & 18,5 \\
\hline Preta & 14 & 21,5 \\
\hline Ignorada & 5 & 7,7 \\
\hline \multicolumn{3}{|l|}{ Escolaridade (em anos) } \\
\hline Nenhuma & 4 & 6,2 \\
\hline 1 a 3 & 8 & 12,3 \\
\hline 4 a 7 & 18 & 27,7 \\
\hline 8 a 11 & 24 & 36,9 \\
\hline$\geq 12$ & 11 & 16,9 \\
\hline
\end{tabular}

Renda (em salários mínimos')

$\begin{array}{lll}\leq 1 & 11 & 16,9\end{array}$

$\begin{array}{lll}2 \text { a } 5 & 28 & 43,9\end{array}$

$\begin{array}{lll}>5 & 11 & 16,9\end{array}$

Ignorada $15 \quad 23,1$

Idade (em anos) (média \pm DP) $\quad 59,1 \pm 14,7$

Morador por domicílio (média \pm DP) $\quad 3,4 \pm 2,9$

'O salário mínimo (SM) vigente na época do estudo era de $\mathrm{R} \$$ 465,00; DP: desvio padrão. 


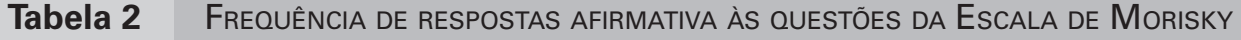

Questões

Sim

$\mathrm{O}(\mathrm{A}) \mathrm{sr}(\mathrm{a})$ alguma vez esquece de tomar os seus medicamentos?

\begin{tabular}{cc}
$\mathrm{n}$ & $\%$ \\
\hline 23 & 35,4 \\
18 & 27,7 \\
4 & 6,2 \\
8 & 12,3
\end{tabular}

Quando o(a) sr(a) está se sentindo melhor, às vezes, para de tomar seus medicamentos?

Algumas vezes, se o(a) sr(a) se sentiu mal, parou de tomar os seus medicamentos?

\section{Tabela 3 Comparação da CLASSIFICAÇÃO dE ADESÃo PELA ESCALA DE MoRISKY COM AS VARIÁVEIS SOCIOECONÔMICAS}

\begin{tabular}{|c|c|c|c|c|c|}
\hline & \multicolumn{4}{|c|}{ Adesão } & \multirow{3}{*}{ Valor $\mathrm{p}$} \\
\hline & \multicolumn{2}{|c|}{ Sim } & \multicolumn{2}{|c|}{ Não } & \\
\hline & $\mathrm{n}$ & $\%$ & $\mathrm{n}$ & $\%$ & \\
\hline Sexo & & & & & 0,718 \\
\hline Feminino & 14 & 48,3 & 19 & 52,8 & \\
\hline Masculino & 15 & 51,7 & 17 & 47,2 & \\
\hline Idade & & & & & 0,001 \\
\hline Até 60 anos & 3 & 10,3 & 14 & 38,9 & \\
\hline Mais de 60 anos & 26 & 89,7 & 22 & 61,1 & \\
\hline Estado civil & & & & & 0,232 \\
\hline Solteiro & 6 & 20,7 & 4 & 11,1 & \\
\hline Casado & 17 & 58,6 & 18 & 50,0 & \\
\hline Viúvo & 4 & 13,8 & 5 & 13,9 & \\
\hline Divorciado & 2 & 6,9 & 9 & 25,0 & \\
\hline Situação de trabalho & & & & & 1,000 \\
\hline Trabalha & 3 & 10,3 & 3 & 8,3 & \\
\hline Não trabalha & 26 & 89,7 & 33 & 91,7 & \\
\hline Cor & & & & & 0,488 \\
\hline Branco & 17 & 58,6 & 17 & 47,2 & \\
\hline Pardo & 4 & 13,8 & 8 & 22,2 & \\
\hline Preto & 5 & 17,2 & 9 & 25,0 & \\
\hline Ignorado & 3 & 10,3 & 2 & 5,6 & \\
\hline Escolaridade & & & & & 0,292 \\
\hline Nenhuma & 2 & 6,9 & 2 & 5,6 & \\
\hline 1 a 3 anos & 1 & 3,4 & 7 & 19,4 & \\
\hline 4 a 7 anos & 7 & 24,1 & 11 & 30,6 & \\
\hline 8 a 11 anos & 13 & 44,8 & 11 & 30,6 & \\
\hline 12 anos ou mais & 6 & 20,7 & 5 & 13,9 & \\
\hline Renda (em salários mínimos) & & & & & 0,052 \\
\hline$\leq 1$ & 2 & 6,9 & 9 & 25,0 & \\
\hline 2 a 5 & 14 & 48,3 & 14 & 38,9 & \\
\hline$>5$ & 3 & 10,3 & 8 & 22,2 & \\
\hline Ignorada & 10 & 34,5 & 5 & 13,9 & \\
\hline Total & 29 & 100,0 & 36 & 100,0 & \\
\hline
\end{tabular}

Nível de significância foi calculado pelo teste Qui-quadrado de independência. Os resultados foram considerados significativos quando $\mathrm{p}<0,05$. 


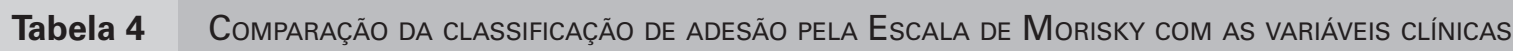

\begin{tabular}{|c|c|c|c|c|c|}
\hline & \multicolumn{4}{|c|}{ Adesão } & \multirow{3}{*}{ Valor $\mathrm{p}$} \\
\hline & \multicolumn{2}{|c|}{ Sim } & \multicolumn{2}{|c|}{ Não } & \\
\hline & $\mathrm{n}$ & $\%$ & $\mathrm{n}$ & $\%$ & \\
\hline $\mathrm{N}^{\circ}$ de medicamentos relatados & & & & & 0,344 \\
\hline Até 3 & 13 & 44,8 & 12 & 33,3 & \\
\hline Mais de 3 & 16 & 55,2 & 24 & 66,7 & \\
\hline $\mathrm{N}^{\circ}$ de medicamentos prescritos & & & & & 0,449 \\
\hline Até 3 & 5 & 17,2 & 9 & 25,0 & \\
\hline Mais de 3 & 24 & 82,8 & 27 & 75,0 & \\
\hline Tempo em acompanhamento & & & & & 0,403 \\
\hline Até 1 ano & 10 & 34,5 & 9 & 25,0 & \\
\hline Mais de 1 ano & 19 & 65,5 & 27 & 75,0 & \\
\hline Diagnóstico etiológico & & & & & 0,501 \\
\hline HAS & 9 & 31,0 & 14 & 38,9 & \\
\hline DM & 9 & 31,0 & 8 & 22,2 & \\
\hline Sem causa definida & 3 & 10,3 & 5 & 13,9 & \\
\hline Rins policísticos & 2 & 6,9 & 2 & 5,6 & \\
\hline Glomerulopatias & 0 & 0 & 3 & 8,3 & \\
\hline Outras & 6 & 20,7 & 4 & 11,1 & \\
\hline Relato de adesão em prontuário & & & & & 0,272 \\
\hline Não & 19 & 65,5 & 28 & 77,8 & \\
\hline Sim & 10 & 34,5 & 8 & 22,2 & \\
\hline Total & 29 & 100,0 & 36 & 100,0 & \\
\hline
\end{tabular}

HAS: hipertensão arterial sistêmica; DM: diabetes mellitus.

Nível de significância foi calculado pelo teste Qui-quadrado de independência. Os resultados foram considerados significativos quando $p<0,05$.

Tabela 5 ComparaÇÃo dA CLASSIFICAÇÃO dE ADESÃo PELA ESCALA DE MORISKY COM AS VARIÁVEIS CLínICO-LABORATORIAIS

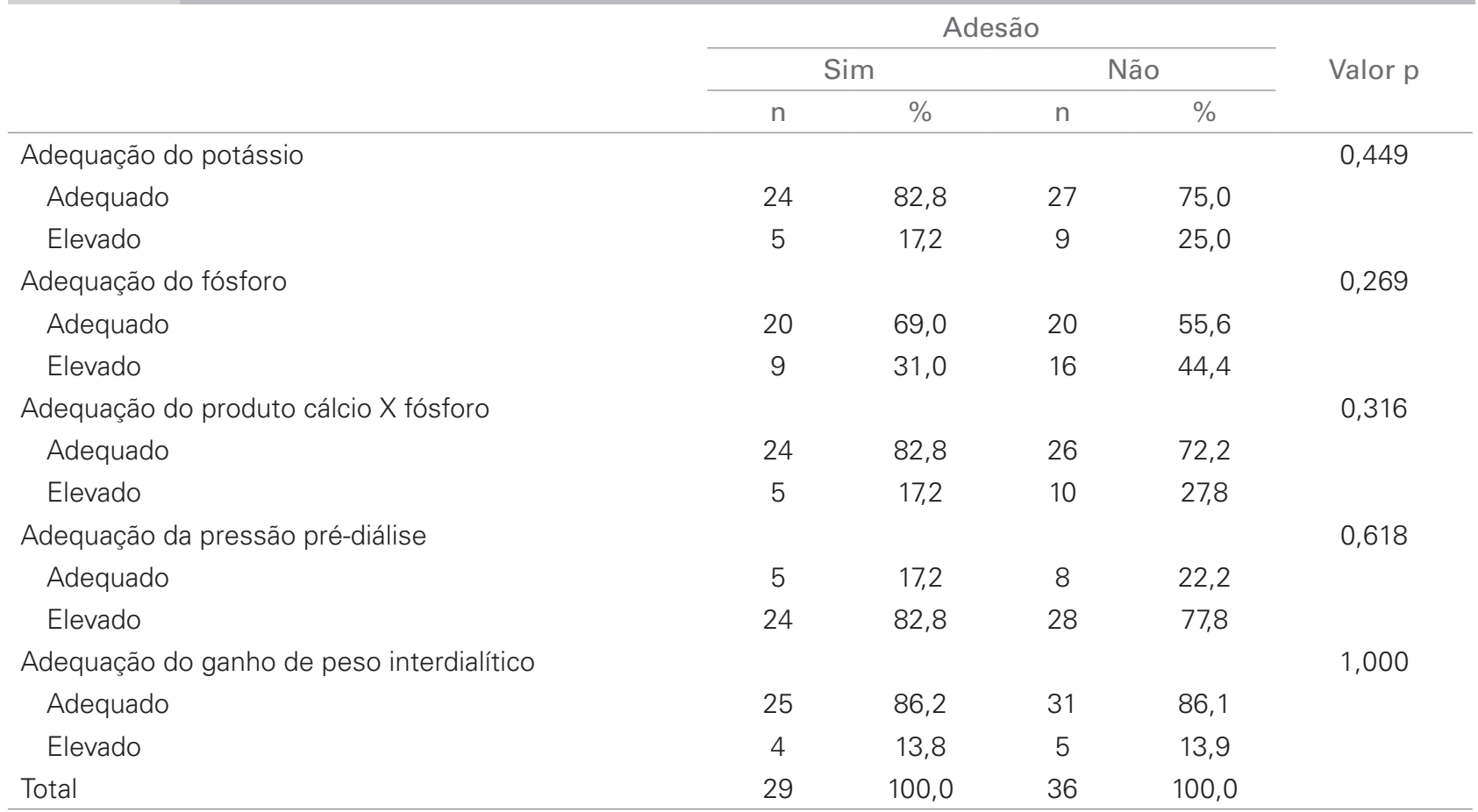

Nível de significância foi calculado pelo teste Qui-quadrado de independência. Os resultados foram considerados significativos quando $p<0,05$. 
aderentes que os adultos jovens $(\mathrm{p}=0,001)$ e o nível salarial, que apresentou-se limítrofe $(\mathrm{p}=0,052)$. Outro dado importante é que no grupo classificado com aderentes, 15 pacientes tinham cuidadores e no grupo de não aderentes, 11 pacientes tinham cuidadores.

Ao analisar somente as variáveis clínico-laboratoriais apresentadas na Tabela 3 e desconsiderando a classificação de adesão, apenas sete $(10,8 \%)$ pacientes foram completamente aderentes a todos os parâmetros.

\section{Dıscussão}

O presente estudo mostrou que a não adesão ao tratamento medicamentoso é frequente nos pacientes em diálise, sendo pior nos pacientes não idosos e em que há um baixo conhecimento sobre os medicamentos.

A não adesão é um achado comum em pacientes com DRC que fazem HD. ${ }^{17}$ Comparando os dados encontrados no presente estudo com resultados de outros trabalhos, a porcentagem estimada de pacientes não aderentes está entre $22-74 \%$ (média $=51 \%$ ). ${ }^{9}, 17,18$ Segundo alguns autores, aproximadamente $50 \%$ dos pacientes podem ser considerados não aderentes em um ou mais aspectos do tratamento. ${ }^{19}$ Deve-se sempre levar em consideração que estudos sobre adesão ao tratamento farmacológico empregam diferentes definições e métodos para mensurá-las, o que torna difícil a comparação dos resultados. ${ }^{8}$ Além disso, a mensuração da adesão ao tratamento por meio das entrevistas apresentou como limitação a superestimativa do número de pacientes considerados aderentes. ${ }^{20}$

Em pacientes adultos que fazem HD, os fatores que podem influenciar na adesão à dieta, medicamentos e restrição de líquidos incluem: idade, cor, sexo, estado civil, nível salarial e escolaridade. ${ }^{17}$ Em diversos estudos, a idade avançada está consistentemente associada ao aumento de adesão, o que também pôde ser constatado no estudo. ${ }^{9,17}$ As possíveis razões que sugerem esse achado são que pessoas idosas são mais preocupadas com a morte e têm uma vida mais estruturada, na qual podem acomodar as demandas de um regime terapêutico. Os pacientes adultos jovens podem ter uma maior dificuldade em aceitar uma condição crônica ou simplesmente são mais propensos a relatar a não aderência quando comparados com os pacientes idosos. ${ }^{17}$

Ao relacionar a escolaridade à adesão, não foi encontrada associação significativa, como também pôde ser constatado em outro estudo. ${ }^{17}$ Em contrapartida, quanto mais baixo o nível de escolaridade maior a probabilidade de abandono ao tratamento, pois a complexidade terapêutica exige dos doentes habilidades cognitivas, muitas vezes não alcançadas por eles. ${ }^{11}$

Em pacientes com doenças crônicas, as taxas de adesão tendem a diminuir com o tempo, com o aumento do número de medicamentos prescritos e com a frequência de doses, os quais podem ser considerados os mais importantes determinantes de adesão. ${ }^{17,21}$ Muitos estudos têm encontrado uma relação inversa entre a frequência de doses prescritas e a adesão, e isso merece uma investigação mais aprofundada, apesar de não ter sido encontrada associação significativa de não adesão relacionada a essas variáveis. ${ }^{21}$ Embora não exista um consenso entre os autores sobre o ponto de corte que deve ser considerado para a comparação entre adesão e número de medicamentos utilizados por cada paciente, no presente estudo foram utilizados como corte três medicamentos, e isso pode ter prejudicado o resultado final pelo fato de que doentes renais em tratamento hemodialítico fazem uso de um grande número de medicamentos.

O efeito negativo da polifarmácia na adesão já é conhecido, pois muitos pacientes não entendem o regime complexo de tratamento e têm dificuldades para organizar seus horários. ${ }^{22} \mathrm{Um}$ tratamento complexo requer do indivíduo uma maior dedicação, seguimento correto das orientações e percepção da importância do tratamento para a manutenção de sua vida. ${ }^{11}$ Dessa forma, a simplificação do esquema terapêutico favorece à adesão, pois facilita a compreensão dos detalhes do tratamento. ${ }^{22} \mathrm{O}$ grande número de medicamentos pode favorecer o não cumprimento do tratamento ou o esquecimento de alguns medicamentos, repercutindo em uma baixa adesão. Apesar de o consumo médio de medicamentos do estudo ter sido de 4,1 $\pm 2,5$ (autorrelato) e 6,2 $\pm 3,0$ (prescrição médica) não ocorreu diferença significativa quando comparado à adesão. No entanto, deve-se considerar que $40 \%$ da amostra foi composta por pacientes que tinham cuidadores responsáveis pela realização do tratamento farmacológico e isso pode ter influenciado negativamente no resultado.

O excessivo ganho de peso interdialítico, a hipertensão arterial e certas condições clínicas identificadas por alterações laboratoriais podem influenciar a adesão ao tratamento. ${ }^{11}$ Além disso, por exemplo, o controle da PA é especialmente importante para adultos com doenças crônicas, pois pode retardar a progressão de algumas doenças e, em outras, ser um sintoma relevante. Para as variáveis clínico-laboratoriais não se achou associação significativa, mas o que pôde ser observado foi o pequeno número de pacientes que apresentaram PA pré-diálise adequada. Segundo um 
estudo, que avaliou 7.890 sessões de HD, considerando como alvo a PA pré-diálise $<140 / 90 \mathrm{mmHg}$, a meta foi alcançada em somente $36 \%$ dos pacientes, e a variação encontrada, entre os 11 centros em que o estudo ocorreu, foi de $28-57 \% .^{15}$

Um ponto interessante e que desempenha papel fundamental na determinação da PA em pacientes em HD é o ganho de peso interdialítico, porém isso não pôde ser constatado no estudo. Alguns autores defendem que considerar apropriado o ganho de peso de até $5 \%$ do peso seco pode ser inadequado e que esse parâmetro deveria ser mais rigoroso para que se pudesse alcançar o controle da PA. ${ }^{23}$

O nível de conhecimento dos pacientes sobre os medicamentos que utilizam é também considerado um fator essencial para a adesão ao tratamento farmacológico. ${ }^{24}$ No presente estudo, apenas uma minoria dos pacientes relataram corretamente o nome e a dose de todos os medicamentos prescritos. Da mesma forma, estudos internacionais avaliaram o grau de conhecimento da terapia farmacológica e encontraram resultados que variam entre 2,5 e $48 \%$ de concordância para a descrição correta do nome dos medicamentos e de 24 e $91 \%$ para o relato correto de suas doses. ${ }^{25}$ Portanto, o desconhecimento dos nomes e das doses dos medicamentos prescritos pode causar erros graves no momento da aquisição e da sua utilização, o que pode levar à inefetividade do tratamento.

A pequena adesão ao tratamento medicamentoso resulta na falha terapêutica e, por esse motivo, existe uma grande preocupação para que o paciente siga o tratamento proposto. A não adesão afeta a qualidade de vida e a sobrevivência a longo prazo do doente renal crônico, levando ao risco de complicações e interferindo no sucesso do tratamento. Desse modo, destaca-se a importância da adesão ao tratamento farmacológico para um melhor controle dos exames laboratoriais, redução das taxas de morbimortalidade e melhoria da qualidade de vida. ${ }^{11}$

\section{Conclusão}

Uma proporção significativa dos pacientes tem dificuldades para aderir ao tratamento medicamentoso e o principal fator citado foi o esquecimento. Das variáveis analisadas, apenas a idade teve relação significativa com o aumento da adesão, sendo os pacientes idosos mais aderentes. Os resultados também sugeriram que os pacientes têm um baixo nível de conhecimento sobre o tratamento farmacológico e esse desconhecimento dos nomes e das doses dos medicamentos prescritos pode ser uma das causas da má adesão. São necessários mais estudos para avaliar a adesão nessa população, a qual necessita adequar a sua vida a uma forma de tratamento tão complexo como a HD e que precisa ter a adesão ao tratamento como um fator essencial para a realização de um plano terapêutico efetivo.

As estratégias para promover a adesão vão também ao encontro da necessidade de melhorias no processo de orientação aos pacientes sobre a DRC e o seu tratamento farmacológico, contemplando toda a equipe de profissionais envolvidos na assistência. O trabalho educativo por meio de grupos com doentes crônicos tem como proposta compartilhar dúvidas, angústias e receios, a fim de buscar alternativas que auxiliem na superação das dificuldades, no enfrentamento e na adaptação do estilo de vida.

\section{RefERÊNCIAS}

1. Sesso R, Gordan P. Dados disponíveis sobre a doença renal crônica no Brasil. J Bras Nefrol 2007;29:9-12.

2. Saran R, Bragg-Gresham JL, Rayner HC, et al. Nonadherence in haemodialysis: associations with mortality, hospitalization, and practice patterns in the DOPPS. Kidney Int 2003;64:254-62.

3. Levey AS, Coresh J, Balk E, et al. National Kidney Foundation Practice Guidelines for Chronic Kidney Disease: evaluation, classification, and stratification. Ann Intern Med 2003;139:137-47.

4. Kara B, Caglar K, Kilic S. Nonadherence with diet and fluid restrictions and perceived social support in patients receiving haemodialysis. J Nurs Scholarsh 2007;39:243-8

5. Kutner NG. Improving conpliance in dialysis patients: does anything work? Semin Dial 2001;14:324-7.

6. Rosner F. Patient noncompliance: causes and solutions. Mt Sinai J Med 2006;73:553-9.

7. Fine RN, Becker Y, De Geest S, et al. Nonadherence Consensus Conference Summary Report. Am J Transplant 2009;9:35-41.

8. Gimenes HT, Zanetti ML, Haas VJ. Factors related to patient adherence to antidiabetic drug therapy. Rev Lat Am Enfermagem 2009;17:46-51.

9. Karamanidou C, Clatworthy J, Weinman J, et al. A systematic review of the prevalence and determinants of nonadherence to phosphate binding medication in patients with end-stage renal disease. BMC Nephrol 2008;9:1-10.

10. Hansen SK. Noncompliance. Nephrol Nurs J 2001;28:653-5.

11. Maldaner CR, Beuter M, Brondani CM, et al. Fatores que influenciam a adesão ao tratamento na doença crônica: o doente em terapia hemodialítica. Rev Gaúcha Enferm 2008;29:647-53.

12. Morisky DE, Green LW, Levine DM. Concurrent and predictive validity of a self-reported measure of medication adherence. Med Care 1986;24:67-74

13. Rocha $\mathrm{CH}$, Oliveira APS, Ferreira $\mathrm{C}$, et al. Adesão à prescrição médica em idosos de Porto Alegre, RS. Ciên Saúde Colet 2008;13:703-10. 
14. Sesso R, Lopes AA, Thomé FS, et al. Relatório do Censo Brasileiro de Diálise, 2008. J Bras Nefrol 2008;30:233-8.

15. Davenport A, Cox C, Thuraisingham R. Achieving blood pressure targets during dialysis improves control but increases intradialytic hypotension. Kidney Int 2008;73:759-64

16. Ifudu O, Uribarri J, Rajwani I, et al. Relation between interdialytic weight gain, body weight and nutrition in haemodialysis patients. Am J Nephrol 2002;22:363-8.

17. Leggat JE Jr, Orzol SM, Hulbert-Shearon TE, et al. Noncompliance in haemodialysis: predictors and survival analysis. Am J Kidney Dis 1998;32:139-45.

18. Gao C, Gruss E, Gonzalez S, et al. Compliance of haemodialysis patients with prescribed medication. J Ren Care 2000;26:4-6.

19. Clearly DJ, Matzke GR, Alexander ACM, et al. Medication knowledge and compliance among patients receiving long-term dialysis. Am J Health Syst Pharm 1995;52:1895-900.
20. World Health Organization (WHO). Adherence to long-term therapies: evidence for action. Geneva: WHO; 2003. p. 198.

21. Paes AH, Bakker A, Soe-Agnie CJ. Impact of dosage frequency on patient compliance. Diabetes Care 1997;20:1512-7.

22. Munger M, Van Tassell BW, Lafleur J. Medication nonadherence: an unrecognized cardiovascular risk factor. MedGenMed 2007;9:58.

23. Bots CP, Brand HS, Veerman ECI, et al. Interdialytic weight gain in patients on haemodialysis is associated with dry mouth and thirst. Kidney Int 2004;66:1662-8.

24. Burge S, White D, Bajorek E, et al. Correlates of medication knowledge and adherence: findings from the residency research network of South Texas. Fam Med 2005;37:712-8.

25. Boonstra E, Lindbaek M, Ngome E, et al. Labelling and patient knowledge of dispensed drugs as quality indicators in primary care in Botswana. Qual Saf Health Care 2003;12:168-75. 